\title{
Novel and Efficient Microwave-Assisted Three Component Reaction for the Synthesis of Oxazine Derivatives
}

\author{
PREETI BANSAL*, NAKUESHWAR DUTT JASUJA and GAJANAND SHARMA \\ Suresh Gyan Vihar University, Jagatpura, Jaipur-302033, India. \\ ${ }^{*}$ Corresponding author E-mail: bpchemistry08@gmail.com
}

http://dx.doi.org/10.13005/ojc/320442

(Received: June 26, 2016; Accepted: July 29, 2016)

\begin{abstract}
Oxazine derivatives can be prepared with yield upto $98 \%$ within a few minutes by an efficient and novel one pot microwave- assisted three-component reaction from 1-naphthol, various anilines and formalin using montmorillonite as the catalyst. The procedure is very simple, efficient and environmentally friendly as it does not use any toxic auxiliary or solvent. The key advantages of this process are high yields, shorter reaction times, and easy work-up and non - chromatographic method has been used for the purification of products.
\end{abstract}

Keywords: Solvent free synthesis, multicomponent reaction, oxazine, microwave.

\section{INTRODUCTION}

Heterocyclic skeletons serve as ideal scaffold on which pharmacophore can be appended to yield potent and special drugs ${ }^{1}$.this is especially true for heterocyclic compound (six member ring), that possess a wide range of interesting biological activities ${ }^{2}$ are core components of a large number of substances. Oxazine derivatives features prominently in many biologically important natural products ${ }^{3}$ and other bioactive molecules ${ }^{4-7}$ the oxazine derivativeshave been used as the basic framework for substance of interest in numerous therapeutic areas such as antifunga ${ }^{8}$ , antibacterial, anti-candina albicans ${ }^{9}$ and kinase inhibitors ${ }^{10}$ In chemistry (sustainable) ${ }^{11}$ the design and development of sequence allowing highly selective essence to determine molecular scaffolds when structural diversity combined ${ }^{12}$ with ecocompatibility ${ }^{13}$ that is great challenges for organic chemists. in a single operation they build one product from three or more reactant molecules with high atom economy ${ }^{14}$ and multiple bond forming efficiency of multiple bond forming ${ }^{15}$. It is their ability. to reach this near ideal goal multicomponent reactions are now well established approaches ${ }^{16}$. Solvent free reactions are of almost interest from the ecological point of view, and they often advantages, such as reduced reaction time, increased product yields, reduced environmental pollution, simple equipment 
(lab scale), increased selectivity and low cost compared with reactions carried out in solvents. As an alternative to organic solvents, chemists should employ other strategies to perform chemical reactions, namely ionic liquid, supercritical fluids, water as solvent and solvent free conditions- like in presence of MW irradiation. This technique does not require solvents and considered "greener" method then the conventional methods. The large scale of applications of microwave chemistry has been increased recently too many aspects of organic synthesis (17-21Microwave assisted synthesis are a particularly attractive alternative to synthesis and severalthermal conditions since they often proceed much faster and synthesis products with higher yields and higher purity.

Although several methods for the preparation of oxazine derivatives have been reported previously ${ }^{22-25}$. Some have been focused on the multicomponent reactions method. This method is advantageous over previous reports due to its short reaction time and solvent free conditions. As per our interest ${ }^{26-28}$ to develop better protocol for the synthesis of biologically active heterocyclic compounds, we would like to report the synthesis of

oxazine derivatives by the reaction of naphthol, formalin, and aniline in presence of MW irradiation.

\section{EXPERIMENTAL METHOD}

from chemical companies Starting material were purchased and used without purification. All microwave assisted were performed by using microwave synthesizer (CEM corp.) (a discover ${ }^{\mathrm{TM}}$ single mode cavity) producing continuous microwave irradiations at $2450 \mathrm{MHz}$ 's. All experiment was conducted under argon. on TLC aluminium roll silica gel $60 \mathrm{~F}_{254}$ (merk) TLC was performed . Melting points weredetermined on a kofler melting point apparatus Melting points were determined. IR spectra were taken on a spectrum one FT-IR spectrometer (Perkin EImer). UV spectra were measured using a CARY 4E spectrophotometer (Varian). NMR spectrawere recorded on a Varian unity INOVA spectrometer (300/ $75 \mathrm{MHz}$ ) in $\mathrm{CDCl}_{3}$; the ${ }^{1} \mathrm{H}$ and $\mathrm{C}^{13}$ chemical shift were referenced to residual solvent signals at $\ddot{a}_{H}$ $=7.25$ and $\ddot{a}_{\mathrm{C}}=77.1$ relative to Tetra methyl silane. Mass spectra were recorded on a MAT 90 with 70 ev ionization energy (finnigan MAT)

\section{EXPERIMENT}

A Mixture of $1 \mathrm{a}(1.0 \mathrm{~m} \mathrm{~mol}), \mathrm{b}(2.0 \mathrm{~m} \mathrm{~mol})$ and $\mathrm{c}(1.0$ $\mathrm{m} \mathrm{mol}$ ) were absorbed on Montmorillonite $(76 \mathrm{mg}$ ) with methanol and mixed thoroughly and irradiated with Microwaves for a particular time till the reaction are completed., the mixture was allowed to cool to r.t. After completion of the reaction. And washed With EE or TBME $(5 X 5 \mathrm{ml})$. In vacuo the combined organic extracts were concentrated And by column chromatography the residue was purified on silica gel to yield (4a)

\section{Spectral data of compounds:-}

(4a) yield- 76\%, mp.-62-63 ${ }^{\mathrm{b}}$, reaction time $-5 \mathrm{~min}$, Anal. Found: C, 82.65\%; H, 5.83\%; N, 5.48\%, Calc.

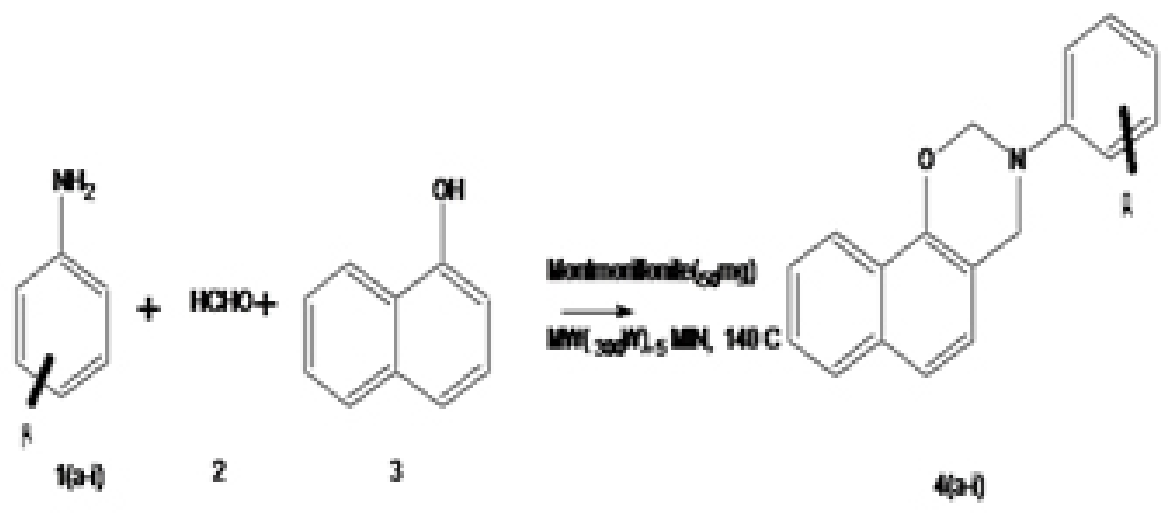

Fig. 1: Microwave assisted three component synthesis of oxazine derivatives 
C18H15NO: C, 82.73; H, 5.79; N, 5.36. IR ( $\mathrm{KBr}$ ímax/cm-1): 1032 (sym.C-O-C), 1213 (asym. C-O-C); 1H NMR (DMSO-d6, 4, $00 \mathrm{MHz}$, ä ppm): 4.78 (s, 2H, -Ar-CH2-N-), 5.42 (s, 2H, - O-CH2-N-), 6.81-7.55 (m, 11H, Ar-H); 13C NMR (DMSO-d6, $75 \mathrm{MHz}$, ä ppm): 49.2, 79.3, 112.6, 115.2, 117.4, 119.7, 120.6, $124.1,125.3,125.3,126.1,127.5,129.1,132.8$, $147.8,148.7$

(4b) yield- $74 \%$, mp. - 300(d), reaction time - $5 \mathrm{~min}$, Anal. Found: C, 78.45; H, 5.90; N, 4.72\%, Calc. C19H17NO2: C, 78.33; H, 5.88; N, 4.81. IR ( $\mathrm{KBr}$, ímax/cm-1): 1018(sym.C-O-C), 1227(asym. C-O-C); 1H NMR (DMSO-d6, $400 \mathrm{MHz}$, ä ppm): 3.61 (s, $3 \mathrm{H}, \mathrm{OMe}), 4.88$ (s, 2H, -Ar-CH2-N-), 5.41 (s, 2H, -O-CH2-N-), 6.78-7.81 (m, 10H, Ar-H); 13C NMR (DMSO-d6, 75MHz, ä ppm): 48.1, 52.1, 80.2, 111.1, $115.5,117.3,119.4,121.2,124.1,125.6,125.8$, $126.7,127.1,130.2,132.2,146.7,148.5$

(4c) Yield- $72 \%$, mp. $-76-77^{\circ}$ c, reaction time- 5 min, Anal. Found: C, 78.71; H, 6.28; N, 4.24, Calc. C2OH19NO2: C, 78.66; H, 6.72; N, 4.59. IR ( $\mathrm{KBr}$, ímax/cm-1): 1027 (sym.C-O-C), 1223 (asym. C-OC);1H NMR (DMSO-d6, $400 \mathrm{MHz}$, ä ppm): 1.21 (t, $3 \mathrm{H}, \mathrm{J}=8 \mathrm{~Hz}, \mathrm{O}-\mathrm{CH} 2-\mathrm{CH} 3$ ), 3.91 (q, 2H, J = $8 \mathrm{~Hz}$ ,O-CH2-CH3), 4.91 (s, 2H, -Ar-CH2-N-), 5.41 (s, 2H, -O-CH2-N-), 6.81-7.81 (m, 10H, Ar-H); 13C NMR (DMSO-d6, 75 MHz, ä ppm): 14.5, 48.3, 65.2, 80.5, $112.5,115.8,117.3,119.2,120.2,123.2,124.7$, $125.3,125.5,126.6,127.8,129.9,132.1,147.3$

(4d) Yield- 76\%, mp. $-72-74$ c, reaction time -5 min, Anal. Found: C, 43.58; H, 2.25; N, 2.64, Caic. : C18H12Br3NO, C: 43.41\%; H: 2.43\%; N: 2.81\%; IR (KBr, ímax/cm-1):1015 (sym.C-O-C), 1225 (asym.
C-O-C); 1H NMR (DMSO-d6, $400 \mathrm{MHz}$, ä ppm): 4.51 (s, 2H, -Ar- CH2-N-), 5.52 (s, 2H, -O-CH2-N-), 6.85-7.91 (m, 8H, Ar-H); 13C NMR (DMSO-d6, 75 $\mathrm{MHz}$, ä ppm):50.1, 79.2, 106.4, 107.8, 119.4, 124.6, $125.3,125.8,126.3,127.1,132.5,133.4,134.8$, $142.7,147.1,150.2$

(4e) Yield- 70\%, mp. - 196-198 c, reaction time - 5 min, Anal. Found: C: $82.71 \%$, H: 6.30\%, N: 5.04\%., Calc.: $\mathrm{C} 19 \mathrm{H} 17 \mathrm{NO}, \mathrm{C}: 82.88 \%$; H: 6.22\%; N: 5.09\%; IR (KBr, ímax/cm-1): 1020 (sym.C-O-C), 1233 (asym. C-O-C); $1 \mathrm{H}$ NMR (DMSO-d6, $400 \mathrm{MHz}$, ä ppm): 2.41 (s, 3H, CH3), 4.91 (s, 2H, -Ar- CH2-N-), 5.61 (s, 2H, -O-CH2- -), 6.61-7.91 (m, 10H, Ar-H); 13C NMR (DMSO-d6, 75 MHz, ä ppm):21.1, 49.1, 78.7, 110.3, $115.1,117.7,119.5,120.2,124.4,125.4,125.8$, $126.2,127.3,129.7,132.2,147.7,148.1$

(4f) Yield - 72\%, mp. - 200(d), reaction time - $5 \mathrm{~min}$, Anal. Found: C: $78.48 \%$, H: 6.37\%, N: 4.67\%., Calc: C2OH19NO2, C: $78.66 \%$; H: 6.27\%; N: 4.59\%; IR (KBr, ímax/cm-1): 1022 (sym.C-O-C), 1235 (asym. C-O-C); 1H NMR (DMSO-d6, $400 \mathrm{MHz}$, ä ppm): 1.28 (t, 3H, J = $14 \mathrm{~Hz}, \mathrm{O}-\mathrm{CH} 2-\mathrm{CH} 3), 3.97$ (q, 2H, $\mathrm{J}=14 \mathrm{~Hz}, \mathrm{O}-\mathrm{CH} 2-\mathrm{CH} 3), 4.62$ (s, 2H, -Ar-CH2-N-), 5.41 (s, 2H, -O-CH2-N- ), 6.18-7.45 (m, 10H, Ar-H); 13C NMR (DMSO-d6, 75 MHz, ä ppm): 13.8, 48.7, 64.6, 81.4, 111.7, 114.6, 115.7, 117.4, 118.7, 121.1, $122.1,124.4,125.5,125.6,126.1,127.5,128.9$, $133.1,146.2,149.2$

(4g) Yield - 74\%, mp. - 118-120 c, reaction time - 5 min, Anal. Found. - C: $77.87 \%$, H: 5.14\%, N: 5.15\%., Calc. C18H14FNO, C: $77.40 \%$; H: 5.05\%; N: 5.01\%; IR (KBr, ímax/cm-1): 1027 (sym.C-O-C), 1246 (asym.

Table 1: Reaction of various aniline with 1- naphthol and formalin (1: 1:2)

\begin{tabular}{|c|c|c|c|c|}
\hline Entry & Products M.F & $\mathbf{R}$ & Reaction time (min) & Mp. $\left({ }^{\circ} \mathrm{C}\right)$ \\
\hline 1 & $\mathrm{C}_{18} \mathrm{H}_{15} \mathrm{NO}$ & $\mathrm{H}$ & $5-10$ & $62-63^{b}$ \\
\hline 2 & $\mathrm{C}_{19} \mathrm{H}_{17} \mathrm{NO}_{2}$ & 4-OMe & $5-10$ & $300(d)$ \\
\hline 3 & $\mathrm{C}_{20} \mathrm{H}_{19} \mathrm{NO}_{2}$ & 4-OEt & $5-10$ & $76-77$ \\
\hline 4 & $\mathrm{C}_{18} \mathrm{H}_{12} \mathrm{Br}_{3} \mathrm{NO}$ & 2,4,6- tri Br & $5-10$ & $72-74$ \\
\hline 5 & $\mathrm{C}_{19} \mathrm{H}_{17} \mathrm{NO}$ & 4- $\mathrm{Me}$ & $5-10$ & 196-198 \\
\hline 6 & $\mathrm{C}_{20} \mathrm{H}_{19} \mathrm{NO}_{2}$ & 2-OEt & $5-10$ & $200(d)$ \\
\hline 7 & $\mathrm{C}_{18} \mathrm{H}_{14} \mathrm{FN}_{\mathrm{O}}$ & $4-\mathrm{F}$ & $5-10$ & $118-120$ \\
\hline 8 & $\mathrm{C}_{19} \mathrm{H}_{17} \mathrm{NO}_{2}$ & 3- OMe & $5-10$ & $280(d)$ \\
\hline 9 & $\mathrm{C}_{19} \mathrm{H}_{17} \mathrm{NO}$ & 2-Me & $5-10$ & $86-88$ \\
\hline
\end{tabular}


BANSAL et al., Orient. J. Chem., Vol. 32(4), 2131-2138 (2016)

2134

Table 2: Synthesis of oxazine derivatives

Entry

1

$\mathrm{H}-\mathrm{NH}_{2}$

2

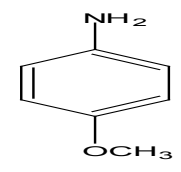

3

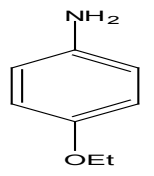

4

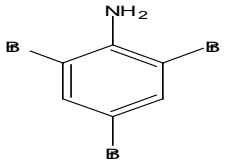

5<smiles>Nc1ccc(Cl)cc1</smiles>

6<smiles>CCOc1ccccc1N</smiles>

7<smiles>Nc1ccc(F)cc1</smiles>

8<smiles>COc1cccc(N)c1</smiles>

9<smiles>Cc1ccccc1N</smiles>

Reactant

(2)

$\mathrm{HCHO}$

$\mathrm{HCHO}$

$\mathrm{HCHO}$
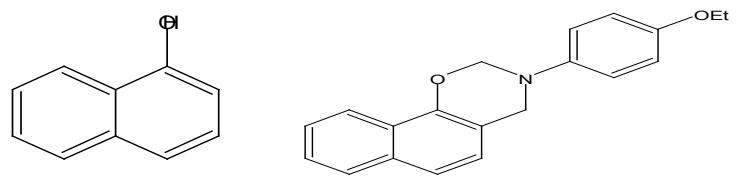

$\mathrm{HCHO}$

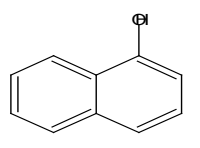

$\mathrm{HCHO}$
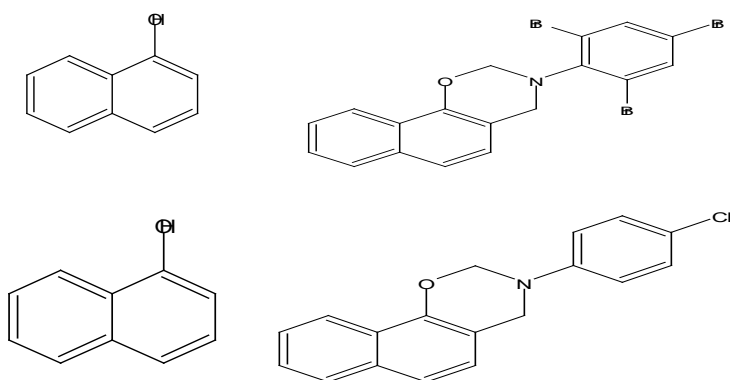

$\mathrm{HCHO}$
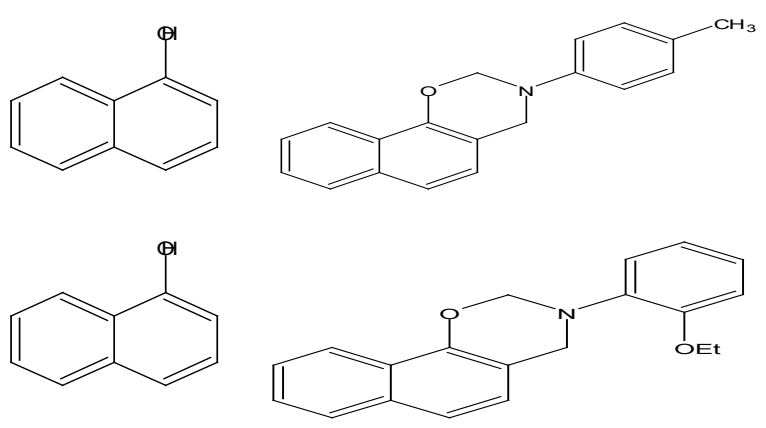

$\mathrm{HCHO}$
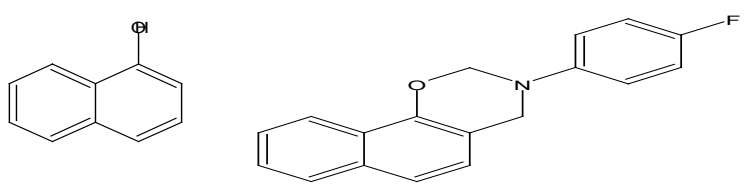

$\mathrm{HCHO}$
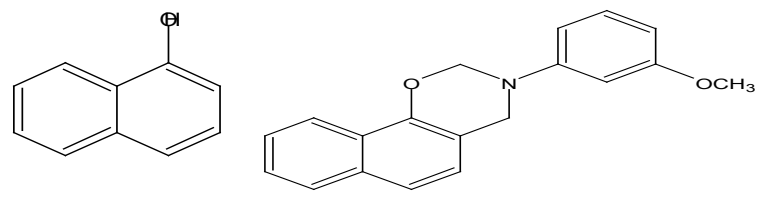

$\mathrm{HCHO}$

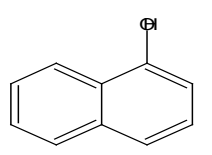<smiles>Cc1ccccc1N1COc2c(ccc3ccccc23)C1</smiles> 
C-O-C);1H NMR (DMSO-d6, $400 \mathrm{MHz}$, ä ppm): 4.91 (s, 2H, -Ar-CH2-N-), 5.61 (s, 2H, -O-CH2-N-), 6.81-7.81 (m, 10H, Ar-H); 13C NMR (DMSO-d6, 75 $\mathrm{MHz}$, ä ppm): 49.1, 78.6, 112.1, 115.6, 116.2, 117.3, $118.1,122.5,123.2,125.2,125.7,125.8,127.6$, $128.5,130.5,150.1$

(4h) Yield - 72\%, mp. -280 (d), reaction time $-5 \mathrm{~min}$, Anal. Found-C: $78.21 \%, \mathrm{H}: 5.78 \%$, N: $4.90 \%$., Calc C19H17NO2, C: $78.33 \%$; H: $5.88 \%$; N: $4.81 \%$; IR (KBr, ímax/cm-1): 1028sym.C-O-C), 1212 (asym. C-O-C); $1 \mathrm{H}$ NMR (DMSO-d6, $400 \mathrm{MHz}$, ä ppm): 3.55 (s, 3H, OMe.), 4.71 (s, 2H, -Ar-CH2-N-), 5.51 (s, 2H, -O-CH2-N-), 6.23-7.64 (m, 10H, Ar-H); 13C NMR (DMSO-d6, 75MHz, ä ppm): 49.4, 53.7, 79.5, $112.3,114.6,115.9,117.4,118.1,120.8,124.6$, $125.7,125.8,126.6,127.6,130.2,133.2,146.8$, $148.8,151.2$ (4i) Yield - 72\%, mp. $-86-88 \mathrm{c}$, reaction time $-5 \mathrm{~min}$, Anal. Found. - C: $82.84 \%$, H: 6.68\%, N: 5.14\%.,Calc C19H17NO, C: $82.88 \%$; H: 6.22\%; N: 5.09\%; IR (KBr, ímax/cm-1): 1028 (sym.C-O-C),1232 (asym. C-O-C); $1 \mathrm{H}$ NMR (DMSO-d6, $400 \mathrm{MHz}$, ä ppm): 2.21(q, 3H, CH3), 4.91 (s, 2H, -Ar- CH2-N-), 5.71 (s, 2H, -O-CH2-N-), 6.81-7.91 (m, 10H, Ar-H); 13C NMR (DMSO-d6, 75 MHz, ä ppm):20.1, 50.2, 79.2, 113.3, 116.2, 117.3, 119.1, 120.4, 124.6, 125.7, $126.0,126.7,127.8,129.6,130.3,147.1,148.2$, $149.1,150.3$

\section{Antibacterial screening}

Prepare Muller Hinton agar medium and put into sterile Petriplates. On agar medium ,200ul of the standard bacterial inoculums was spread by using sterile cotton swab. In the inoculated agar medium the test impregnated discs were placed. To determine

Table 3: Antibacterial and Antifungal Activity of Compounds (Newly Synthesized Oxazine Derivatives (1a-1i))

\begin{tabular}{|c|c|c|c|c|}
\hline \multicolumn{5}{|c|}{ Antibacterial activity } \\
\hline Compound10 $\mu \mathrm{g} / \mathrm{ml}$ & S.aureus & B.subtillus & E.Coli & P. Aeruginosa \\
\hline $1(\mathrm{a})$ & 10 & 12 & 8 & 6 \\
\hline $1(b)$ & 15 & 19 & 17 & 9 \\
\hline $1(\mathrm{c})$ & 17 & 21 & 18 & 9 \\
\hline $1(d)$ & 18 & 17 & 25 & 21 \\
\hline $1(\mathrm{e})$ & 11 & 7 & 5 & 8 \\
\hline $1(f)$ & 15 & 18 & 19 & 8 \\
\hline $1(\mathrm{~g})$ & 16 & 13 & 21 & 17 \\
\hline $1(\mathrm{~h})$ & 13 & 15 & 18 & 10 \\
\hline 1 (i) & 9 & 8 & 6 & 7 \\
\hline ciprofloxacin & 19 & 22 & 26 & 22 \\
\hline \multicolumn{5}{|c|}{ Antifungal activity } \\
\hline Compound $10 \mu \mathrm{g} / \mathrm{ml}$ & C. Albicans & A.niger $C$ & Chrysosporium sp. & Trichoderma sp. \\
\hline $1 a$ & 9 & 8 & 7 & 6 \\
\hline $1 b$ & 17 & 15 & 14 & 13 \\
\hline $1 \mathrm{c}$ & 18 & 17 & 19 & 16 \\
\hline $1 d$ & 10 & 12 & 13 & 14 \\
\hline $1 e$ & 7 & 9 & 8 & 6 \\
\hline $1 f$ & 17 & 15 & 16 & 14 \\
\hline $1 \mathrm{~g}$ & 7 & 8 & 9 & 8 \\
\hline $1 \mathrm{~h}$ & 16 & 13 & 15 & 12 \\
\hline $1 \mathrm{i}$ & 8 & 7 & 9 & 6 \\
\hline Clomatrimazole & 19 & 18 & 20 & 18 \\
\hline
\end{tabular}


Table 4: Antifungal activity of compounds (newly synthesized oxazine derivatives (1a-1i))

\begin{tabular}{lcccc}
\hline Compound 10 $\mathbf{H g} / \mathbf{m l}$ & C. Albicans & A.niger & Chrysosporium sp. & Trichoderma sp. \\
\hline 1a & 9 & 8 & 7 & 6 \\
1b & 17 & 15 & 14 & 13 \\
1c & 18 & 17 & 19 & 16 \\
1d & 10 & 12 & 13 & 14 \\
1e & 7 & 9 & 8 & 6 \\
1f & 17 & 15 & 16 & 14 \\
1g & 7 & 8 & 9 & 8 \\
1h & 16 & 13 & 15 & 12 \\
1i & 8 & 7 & 9 & 6 \\
Clomatrimazole & 19 & 18 & 20 & 18 \\
\hline
\end{tabular}

the sensitivity of each microbial species tested Ciprofloxacin $10 \mathrm{ug} / \mathrm{ml}$ capacity discs were used as positive references standard. For 24 hours all petriplates were incubated at $37^{\circ} \mathrm{C}$. Diameter of inhibition was measured after incubation

\section{Antifungal screening}

Prepared a Sabouraud dextrose agar medium and transferred into sterile petriplates. On agar medium $200 \mathrm{ul}$ of the standardized fungal inoculums were spread by using cotton swab. On the inoculated agar medium the test impregnated discs were placed.To determine sensitivity of each microbial species tested Clomatrimazole $10 \mathrm{ug} / \mathrm{ml}$ was used as positive references standard. For 24 hours all petriplates were incubated at $37^{\circ} \mathrm{C}$. diameter of zone of inhibition was measured After the incubation

\section{RESULT AND DISCUSSION}

Our results is present on the microwave - assisted three component synthesis of oxazine derivatives. When amounts of 1 (a) aniline, 2(a) 1 naphthol, 3(a) formalin were reacted in the presence of montmorillonite in a sealed via under microwave conditions. A focused single mode microwave reactor for $5 \mathrm{~min}$ at $140^{\circ} \mathrm{C}$. has been used.Under these conditions [1,3] oxazine derivatives $4(a)$ could be isolated app. In $76 \%$ yield.

\section{Antibacterial screening}

The bacterial inhibition values $(\mathrm{mm})$ are shown in table-3. The antimicrobial activities of compounds S. Aureus, Escherichia Coli, Bacillus Subtillus and P. Aeruginosa were screened. Ciprofloxacin were used as a standard at $100 \mathrm{ug} /$ $\mathrm{ml}$. Compound 1a-1i were screened. S. Aureus for compound $1 \mathrm{~d}$ was found to be highly active on the other hand for other compounds had low activity with the ciprofloxacin. B. Subtillus shows highly activity for compound $1 \mathrm{c}$, on the other hand for other compoundsshows low activity with the standard ciprofloxacin. E. coli for compound 1d was found to be highly active, on the other hand for other compounds had low activity compared with the standard ciprofloxacin. P. Aeruginosa for compound $1 \mathrm{~d}$ was found to be highly active, on the other hand for other compounds compound had low activity in comparison the standard ciprofloxacin

\section{Antifungal screening}

The fungal inhibition zone values $(\mathrm{mm})$ are given in table-4. The antifungal activity of compounds C. Albicans, Aspergillus Niger, Chrysosporium sp., Trichoderma sp. Were screened. At a $100 \mathrm{\mu gmL}^{-1}$ Clomatrimazole were used as a standard.Compound 1a-1i were screened. C. Albicans for compound 1b and $1 f$ was found to be Highly active, on the other hand for compound 1a, 1c, 1d, 1e, 1g, 1h, 1i, had low activity compared with standard Clomatrimazole.

Aspergillus Niger for compound 1c was found to be highly active on the other hand for compound 1a, 1b, 1d, 1e, 1f, 1g, 1h, 1i, had low activity compared with standard Clomatrimazole. Chrysosporium sp. For compound 1c was found 
to be highly active On the other hand for $1 \mathrm{a}, 1 \mathrm{~b}, 1 \mathrm{~d}$, 1e, 1f, 1g, 1h, 1i show low activity with standard Clomatrimazole. Trichoderma sp. for compound 1c was found to be highly active, On the other hand for $1 \mathrm{a}, 1 \mathrm{~b}, 1 \mathrm{~d}, 1 \mathrm{e}, 1 \mathrm{f}, 1 \mathrm{~g}, 1 \mathrm{~h}, 1 \mathrm{i}$ show low activity with standard Clomatrimazole.

\section{CONCLUSION}

we have designed an environmentally friendly, green and efficient approach for the synthesis of oxazine derivatives. By solvent free, the microwave assisted three component reactions with yield up to $76 \%$ within a few minutes. The method is important due to high conversion, less reaction time, and clean reaction profile, simple experimental and work-up procedure.

\section{ACKNOWLEDGMENT}

The author would like to thank the head, department of chemistry, Suresh gyan Vihar University, Jaipur (raj.) for the financial support. $x z$ and providing necessary laboratory facilities.

\section{REFERANCES}

1. Hermakens, P.H.H.; Ottenheijm, H.C.J.; Rees, D.C. Tetrahedron 1997, 53, 5643-5678; (b) Gordon, E.M.; Barrett, R.W.;Dower, W.J.; Folder, S.P.A ; Gallop, M.A.J. Med. Chem. 1994, 37, 1385-1401

2. Avendano, C.; Menendez, J.C. Medicinal chemistry of Anticancer Drugs; Elsevier, 2008; (b) Doherty, A.M. Annual Reports in medicinal chemistry; Academic Press 2000; 35, (c) Patrick, G.L. An introduction to medicinal chemistry; Oxford University; 1995

3. Domling, A., Recent developments in isocyanide based multicomponent reactions in applied Chemistry. Chem.Rev. 2006, 106, 17-89.

4. Kobayashi, M.J. Kitazawa, M. ; Sotio , T.; Yamamoto, R.; Harada, H. Studies on the synthesis of antiulcer agents. II. Synthesis and antiulcer activity of cyclic carbamate derivatives. Yakugaku Zasshi 1984, 104, 659679; chem. Abstr. 1985, 102, 6344 m.

5. Testa , E,; Fontanella , L.; Cristiahi, G.; Gallo, G. 5,5 - Disub stituted dihydro-1,3- oxazine $-2,4$ - diones, research on compounds active on central nervous system XII. J.Org. Chem. 1959. 24. 1928-1936.

6. Vrouenraets, S.M.;Wit, W.F.; Van Tongeren, J.; Lange, J.M. Efavirenz; a review, Expert, Opin, pharmacother. 2007. 8. 851-871.

7. Fauran , C.P.; Douzon, C.; Raynaud, G.; Sergant, M.; Novel derivatives of substituted tetra hydro M-Oxazines, their process of preparation and their therapeutic application., US 3, 821, 215, 1974; Chem. Abstr. 1974, 82,
125412

8. Fringuelli, R.; Pietrella, D.; Schiaffella, F.; Guarra, I.A.; Perito, S; Bistoni, F.; Vecchiarelli, A.; Biorg. Med. Chem. 2002, 10, 1681-1686

9. Macchiarulo, A.; Costantino, G.; Fringuelli, f.; Vecchiarelli, A.; Schiaffella, F.; Fringuelli, R. Bioorg. Med. Chem. 2002, 10, 3415-3423

10. Adans, N.D.; Darcy, M.G.; Dhanek, D.; Duffy, K.J.; Fitch, D.M.; Knight, S.D.; Newlander, K.A.; A.N. Int. Patent. Wo 2006, 113432, 2006; Chem. Abstr. 2006, 145, 438652

11. Special issue in Green chemistry, See: Chem. Rev., 2007, 107, 2167

12. Schreiber,S.L.; Nature, 2009, 457, 153.

13. We define eco-compatible as both economically and ecologically compatible

14. Trost, B.M. ; Acc. Chem. Res.; 2002, 35, 695

15. Wender, P.A. ; Verma, U.A. ;. Paxton, T.J ; and. Pillow, T.H; Acc. Chem. Res., 2008, 41, 40

16. Zhu, ed. J++ and Bienayme, H.; Wiley -VCH, Multicomponent reactions, Weinheim , 2005

17. Wender, P.A.; Handy, S.T.; And. Wright, D.L; Chem. Ind., 1997, 765

18. De Simore, J.M.; Practical approaches to green solvents. Science, 2002, 297, 799803

19. Sharma J, Chin B, Huibers PDT, et al. Solvent replacement for green processing. Environ. Health perspect., 1998, 106 (Suppl.1): 253271,. 20. Roberts B A, Strauss CR. Towards rapid, "green" predictable microwave-assisted synthesis. Review. Acc. Chem. Res., 2005, 38, 653-661

21. Larhed M, Moberg C, Hallberg A. Microwave 
accelerated homogeneous catalysis in Organic chemistry. Acc. chem. Res., 2002, 35, 717-727,

22. Caddick S.; Microwave assisted organic reactions. Tetrahedron ; 1995, 51, 1040310432,

23. Agag, T.; Preparation and properties of some thermosets derived from allyl-functional naphtooxazines. J. App. Poly. Sci. 2006, 100, 3769-3777

24. Burke W.J.; Murdock, K.C.; Ec, G.; Condensation of hydroxy aromatic compounds with formaldehyde and primary aromatic amines. J. Am. Chem. Soc. 1954, 76, 16771679

25. Mathew, B.P.; Nath, M.; One pot three component synthesis of dihydrobenzo and naphtho [e] - 1, 3 -oxazines in water. J.
Heterocyclic Chem. 2009, 46, 1003-1006

26. Sapkal, S.B.; Shelke, K.F.; Shingate, B.B.; Shingare, M.S. ; Nickel nanoparticle catalyzed facile and efficient one pot synthesis of polyhydroquinoline derivatives via Hantzsch condensation under solvent free conditions. Tetrahedron Lett. 2009, 50, 1754-1756.

27. Sonar, S.S ; Kategaonkar, A.H.; Ware, M.N.; Grill, C.H.; Shingate, B.B.; Shingare, M.S.; Ammonium metavanadate:an effective catalyst for synthesis of á- hydroxyphosphonates. Arkivoc 2009, ii, 138-148.

28. Sonar, S.S.; Sadaphal, S.A.; Kategaonkar, A.H.; Pokalwar, R.U.; Shingate, B.B.; Shingare, M.S.; Alum Catalysed Simple and efficient synthesis of bis (indolyl) methanes by Ultrasound Approach. Bull. Korean Chem. Soc. 2009, 30, 825-828. 